\title{
Entropy Conservation of Linear Dilaton Black Holes in Quantum Corrected Hawking Radiation
}

\author{
I.Sakalli.* H.Pasaoglu $\doteq$ and M. Halilsoy: \\ Department of Physics, Eastern Mediterranean University, \\ G. Magusa, North Cyprus, Mersin-10, Turkey.
}

\begin{abstract}
It has been shown recently that information is lost in the Hawking radiation of the linear dilaton black holes in various theories when applying the tunneling formulism without considering quantum gravity effects. In this Letter, we recalculate the emission probability by taking into account of the log-area correction to the Bekenstein-Hawking entropy and the statistical correlation between quanta emitted. The crucial role of the black hole remnant on the entropy conservation is highlighted. We model the remnant as a higher dimensional linear dilaton vacuum in order to show that such a remnant model cannot radiate and its temperature would be zero. In addition to this, the entropy conservation in the higher dimensional linear dilaton black holes is also discussed. In summary, we show in detail that the information can also leak out from the linear dilaton black holes together with preserving unitarity in quantum mechanics.
\end{abstract}

\footnotetext{
*Electronic address: izzet.sakalli@emu.edu.tr

${ }^{\dagger}$ Electronic address: hale.pasaoglu@emu.edu.tr

${ }^{\ddagger}$ Electronic address: mustafa.halilsoy@emu.edu.tr
} 


\section{INTRODUCTION}

Since the seminal works of Bekenstein [1] and Hawking [2], it is known that a black hole $(\mathrm{BH})$ behaves as a thermodynamic system, radiating as a black body with characteristic temperature and entropy. Today, the thermodynamic properties of BHs are well understood such that there are numerous independent derivations that all give the same quantitative results: Hawking temperature $T_{H}=\frac{\kappa}{2 \pi}$ and Bekenstein-Hawking entropy $S_{B H}=\frac{A_{h}}{4}$, where $\kappa$ is the surface gravity and $A_{h}$ is the horizon area of the BH. However, in today's physics Hawking's original derivation is considered only a skillful application of quantum field theory in curved spacetime. Although it is not directly connected to quantum gravity, nevertheless it has a strong impact on quantum-gravitational problems toward understanding the statistical origin of the $\mathrm{BH}$ entropy, and its natural consequence - information concept [3] in the BHs. With the traditional picture of the Hawking radiation, which generates entangled pairs and the state of the outgoing quanta is a mixed (pure thermal radiation) when the BH completely evaporates, one encounters with two serious and simultaneous problems. One of them is the violation of the unitarity in quantum mechanics (QM), which does not allow any evolution process from a pure quantum state into a mixed state. The second is the information loss about the original quantum state that formed the black hole. The latter phenomenon is called "information loss paradox" [4]. In order to resolve this paradox, one finds many attempts in the literature, see for instance [5, 6] (and references therein). Among them, tunneling of emitted particles through the horizon is one of the popular methods. The speech-ripe idea of the method dates back to 1999 [7], but the nicest form was developed a decade ago by Parikh and Wilczek [8]. In a very short time, Parikh and Wilczek's tunneling method has been used and improved further by other researchers [9], and it has been known as the tunneling formulism. This formulism has recently been improved one level up by Chen and Shao [6] by considering the statistical correlations, which exist among the emitted quanta [10]. In [6], it has successfully been shown that emitted particles would leak out information from the BH. Results of [6] do not only resolve the information loss paradox, but preserve total entropy conservation and the unitarity of QM as well. All those impressive results in [6] have become possible with the inclusion of the quantum gravity corrections and the existence of $\mathrm{BH}$ remnant.

Study [6] has given us a hope also to resolve the information loss problem in our recent 
study [11], which has explored the Hawking radiation of linear dilaton black holes (LDBHs) within the context of Maxwell, Yang-Mills and Born-Infeld theories. In [11], we have not considered the BH remnant with quantum corrections. Our calculations yield a pure thermal spectrum, which corresponds to the violation of the unitarity in QM and signals the information loss in the Hawking radiation of the LDBHs.

In this Letter, following [6] as a guide, we calculate the correlation between successively emitted quanta from the LDBHs. The existence of the LDBH remnant in preserving entropy conservation will be much more important than the Schwarzschild case [6]. In Section 2, we apply the tunneling formulism to the LDBHs in order to obtain the quantum gravity corrected BH entropy and the emission probability. Section 3 is devoted to the calculation of the correlation between two successively emitted quanta. In the following Section, complete radiation process is considered in which particles are successively emitted from the LDBH until reaching the remnant with an emphasis on the entropy conservation. Similarly, in Section 5, we consider the case of higher dimensional LDBHs [12]. First, we model the remnant as a higher dimensional linear dilaton vacuum and show, from the absence of reflection, that its temperature must be zero. Secondly, we reconsider the generic higher dimensional LDBHs and calculate the entropy of the pointlike remnant BH. We draw our conclusions in Section 6.

Throughout the Letter, the units $G=c=k_{B}=\hbar=1$ and the Planck length $L_{p}=$ $\sqrt{\frac{\hbar G}{c^{3}}}=1$ are used.

\section{TUNNELING RATE WITH QUANTUM CORRECTIONS FOR $4 D$-LDBHS}

As it can be seen from [11], 4D-LDBHs in Einstein-Maxwell-Dilaton (EMD), EinsteinYang-Mills-Dilaton (EYMD) and Einstein-Yang-Mills-Born-Infeld-Dilaton (EYMBID) theories are described by the line element.

$$
d s^{2}=-f d t_{L}^{2}+\frac{d r^{2}}{f}+R^{2} d \Omega_{2}^{2}
$$

where $t_{L}$ is the LDBH time and $d \Omega_{2}^{2}=d \theta^{2}+\sin ^{2} \theta d \phi^{2}$. Here, the metric functions are given by

$$
f=\Sigma r\left(1-\frac{r_{+}}{r}\right) \quad \text { and } \quad R=A \sqrt{r}
$$


It is obvious that metric (1) represents a static non-rotating $\mathrm{BH}$ with a horizon at $r_{+}$. The coefficients $\Sigma$ and $A$ in the metric functions take different values according to the concerned theory (EMD, EYMD or EYMBID) [11]. Furthermore, it is a non-asymptotically flat (NAF) spacetime. For $r_{+} \neq 0$, the horizon hides the null singularity at $r=0$. However, in the extreme case $r_{+}=0$, the central null singularity $r=0$ is marginally trapped in which it does not allow outgoing signals to reach external observers. Namely, even in the extreme case of $r_{+}=0$ metric (1) maintains its black hole property.

By using the definition of quasi-local mass $M$ [13] for our NAF metric (1), one can get a relationship between the horizon $r_{+}$and the mass $M$ as follows

$$
r_{+}=\frac{4 M}{\Sigma A^{2}}
$$

Since none of the curvature invariants of metric (1) are singular on the horizon $r_{+}$, one would transform to a new coordinate system, which can be a non-singular at $r_{+}$. For this purpose, we pass to Painlevé-Gullstrand type coordinates

$$
d s^{2}=-f(r) d t^{2}+2 \sqrt{1-f(r)} d t d r+d r^{2}+R^{2} d \Omega^{2}
$$

with

$$
d t=d t_{L}+\frac{\sqrt{1-f(r)}}{f(r)} d r
$$

where metric (4) has a number of advantages for such problems. Of course the curvature singularity at the origin $r=0$ is still present in both coordinate systems (1) and (4). Meanwhile, from the Schwarzschild problem it is known that in these coordinates the time $t$ is linearly related to the proper time for a radially falling observer. Obviously, the hypersurfaces $t=$ const. are all intrisically flat.

In metric (4), the radial null geodesics of a test particle considered as a massless spherical shell have a rather simple form,

$$
\dot{r}=\frac{d r}{d t}=-\sqrt{1-f(r)} \pm 1
$$

where the choice of signs depends on whether the rays are outgoing $(+)$ or ingoing $(-)$. In Painlevé-Gullstrand coordinates (4), the surface gravity on the horizon, which is the function of the $\mathrm{BH}$ mass $M$ is one of the Christoffel components. 


$$
\kappa(M)=\Gamma_{00}^{0}=\frac{1}{2} f^{\prime}\left(r_{+}\right),
$$

Since the metric function $f$ is zero on the horizon, it can be expanded around $r_{+}$as

$$
f=f^{\prime}\left(r_{+}\right)\left(r-r_{+}\right)+O\left(r-r_{+}\right)^{2},
$$

By virtue of the $4 D$-LDBHs the $O\left(r-r_{+}\right)^{2}$ vanishes, and we have an exact expression. However, in the higher dimensional cases, which we consider in Section V, an expression around $r_{+}$will be used. Therefore, near the horizon of the $4 D$-LDBH, the radial outgoing null geodesics can be expressed as

$$
\dot{r}=\frac{d r}{d t}=\frac{1}{2} f^{\prime}\left(r_{+}\right)\left(r-r_{+}\right)
$$

which is nothing but

$$
\dot{r}=\left(r-r_{+}\right) \kappa(M)
$$

Let us consider a spherically symmetric system whose total mass $M$ is kept fixed. We assume that the system consists of a LDBH with varying mass $M-\omega$, emitting a spherical shell of mass $\omega$ such that $\omega \ll M$. This phenomena is known as self-gravitation effect [14]. After taking into account of such self-gravitation effects, $\dot{r}$ can be rewritten as

$$
\dot{r}=\left(r-r_{+}\right) \kappa(M-\omega),
$$

In the WKB approximation, the tunneling rate for an outgoing positive energy particle, which crosses the horizon from $r_{\text {in }}$ to $r_{\text {out }}$, is related to the imaginary part of the particle's action [8, 14] in accordance with

$$
\Gamma \sim e^{-2 \operatorname{Im}(I)}
$$

Imaginary part of the particle's action is calculated from

$$
\operatorname{Im}(I)=\operatorname{Im} \int_{r_{\text {in }}}^{r_{\text {out }}} p_{r} d r=\operatorname{Im} \int_{r_{\text {in }}}^{r_{\text {out }}} \int_{0}^{p_{r}} d \tilde{p}_{r} d r,
$$

Using Hamilton's equation for the classical trajectory in the form 


$$
d p_{r}=\frac{d H}{\dot{r}},
$$

with $H=M-\omega$ i.e. $d H=-d \omega$, and inserting $\dot{r}$ given by Eq. (10) into Eq. (13), one gets

$$
\operatorname{Im}(I)=-\operatorname{Im} \int_{r_{i n}}^{r_{o u t}} \int_{0}^{\omega} \frac{d \tilde{\omega}}{\left(r-r_{+}\right) \kappa(M-\tilde{\omega})} d r
$$

One can evaluate the integral over $r$ by deforming a contour, where its semicircle centered at real axis pole $r_{+}$. Thus we get

$$
\operatorname{Im}(I)=-\pi \int_{0}^{\omega} \frac{d \tilde{\omega}}{\kappa(M-\tilde{\omega})},
$$

The reason of the sign change in (15) is due to the fact that the horizon will shrink during the process of Hawking Radiation, $r_{\text {out }}<r_{i n}$. In other words, the horizon tunnels inward as the BH's mass decreases.

According to corrections of surface gravity, the quantum gravity surface gravity [10, 15] is defined as

$$
\kappa_{Q G}=\kappa(M-\omega)
$$

and Eq. (16) becomes

$$
\operatorname{Im}(I)=-\pi \int_{0}^{\omega} \frac{d \tilde{\omega}}{\kappa_{Q G}},
$$

Hawking temperature is still expressed in the form $T_{H}=\frac{\kappa_{Q G}}{2 \pi}$. By using the Hawking temperature in Eq. (18), we find

$$
\begin{aligned}
\operatorname{Im}(I) & =-\frac{1}{2} \int_{0}^{\omega} \frac{d \tilde{\omega}}{T_{H}}=-\frac{1}{2} \int_{S_{\text {in }}}^{S_{\text {out }}} d S \\
& =-\frac{1}{2} \int_{S_{Q G}(M)}^{S_{Q G}(M-\omega)} d S \\
& =\frac{1}{2}\left[S_{Q G}(M)-S_{Q G}(M-\omega)\right] \\
& =\frac{1}{2} \Delta S_{Q G} .
\end{aligned}
$$


where $S_{Q G}$ is the corrected area entropy for BH. In string theory and loop quantum gravity [16] it is introduced with a logarithmic correction

$$
S_{Q G}=\frac{A_{h}}{4}+\alpha \ln A_{h}+O\left(\frac{1}{A_{h}}\right),
$$

where $\alpha$ is a dimensionless constant. It takes different values according to which theory is considered. The other physical parameter $A_{h}$ is the area of horizon $A_{h}$. For the $4 D$-LDBHs, $A_{h}=4 \pi A^{2} r_{+}=16 \pi \frac{M}{\Sigma}$, and one easily reads the tunneling rate with quantum correction as

$$
\Gamma \sim e^{-2 \operatorname{Im}(I)}=e^{-\Delta S_{Q G}}=\left(1-\frac{\omega}{M}\right)^{\alpha} \exp \left(-4 \pi \frac{\omega}{\Sigma}\right) .
$$

Here, $\left(1-\frac{\omega}{M}\right)^{\alpha}$ is an additional factor compared with the previous tunneling rate in [11]. This expression comes from quantum gravitational effects on the energy of emitted particles and the mass of the LDBH. In other words, it is natively due to the effect of back reaction. As it will be seen in the following sections, it will play a great role on entropy conservation as well as on the information loss paradox. One may notice that the coefficient of power $\alpha$ herein is 1, as distinct from 2 appeared in the tunneling calculations of Schwarschild black hole, see for instance [6].

\section{STATISTICAL CORRELATION BETWEEN TWO SUCCESSIVE EMIS- SIONS}

According to quantum gravity effects and its consequence (21), contrary to the classical tunneling method employed in [11], we cannot consider anymore the LDBHs as objects emitting only pure thermal radiation. From physical point of view, the deviation (21) from thermal spectrum is very important, and it may cause some therapeutic effects on the information loss appeared in the LDBHs [11]. In this section, we shall check whether or not the emission probabilities for two successive modes are statistically correlated [17]. Similar to the conclusion of non-trivial correlation obtained in [6], we expect to find statistically dependent correlations between two successive emissions. By that way, we would like to open a gate in order to resolve the information paradox in the LDBHs. Throughout this section, we shall follow the method employed in [6]. 
Firstly, we consider initially two successive emissions, with energies $\omega_{1}$ and $\omega_{2}$. Using Eq. (21), for the first emission of energy $\omega_{1}$ we have

$$
\Gamma\left(\omega_{1}\right)=\left(1-\frac{\omega_{1}}{M}\right)^{\alpha} \exp \left(-4 \pi \frac{\omega_{1}}{\Sigma}\right),
$$

Then a second emission of energy $\omega_{2}$ on the condition that the first one $\omega_{1}$ is

$$
\Gamma\left(\omega_{2} \mid \omega_{1}\right)=\left(1-\frac{\omega_{2}}{M-\omega_{1}}\right)^{\alpha} \exp \left(-4 \pi \frac{\omega_{2}}{\Sigma}\right),
$$

which is known as the conditional probability [10]. Removing the condition on the second emission, we get

$$
\Gamma\left(\omega_{2}\right)=\left(1-\frac{\omega_{2}}{M}\right)^{\alpha} \exp \left(-4 \pi \frac{\omega_{2}}{\Sigma}\right)
$$

which is the probability just for the second emission. The emission of the total energy yields

$$
\Gamma\left(\omega_{1}+\omega_{2}\right)=\left(1-\frac{\omega_{1}+\omega_{2}}{M}\right)^{\alpha} \exp \left(-4 \pi \frac{\omega_{1}+\omega_{2}}{\Sigma}\right),
$$

The statistical correlation between two successive emissions is measured by [18]

$$
\chi\left(\omega_{1}+\omega_{2} ; \omega_{1}, \omega_{2}\right)=\ln \frac{\Gamma\left(\omega_{1}+\omega_{2}\right)}{\Gamma\left(\omega_{1}\right) \Gamma\left(\omega_{2}\right)},
$$

which is calculated as

$$
\chi\left(\omega_{1}+\omega_{2} ; \omega_{1}, \omega_{2}\right)=\alpha \ln \left(1-\frac{\omega_{1} \omega_{2}}{\left(M-\omega_{1}\right)\left(M-\omega_{2}\right)}\right) .
$$

First of all, it is evident that the correlation strictly depends on $\alpha$. In the Schwarschild $\mathrm{BH}$ [6], even in the case of $\alpha=0$, the correlation is non-zero. But, here once $\alpha=0$ is set to zero the subsequent emissions become statistically independent, and thus information paradox might never be resolved. Essentially, in the absence of $\alpha$ the result (27) explains why we have not obtained any deviation from pure thermal spectrum in our recent study [11]. However, the case $\alpha \neq 0$ show that the successive emissions are statistically dependent, which means that there must be correlations between the successive emissions. In summary, Eq. (27) indicates that information should leak out from the LDBHs during their radiation. 


\section{ENTROPY CONSERVATION AND BH REMNANT}

It is known that calculation of entropy carried by Hawking radiation is obtained best by considering the complete process of the $\mathrm{BH}$ evaporation. For this purpose, we consider the emission of $n$ particles with energies $\omega_{1}, \omega_{2}, \ldots \ldots, \omega_{n}$, which are successively emitted from the LDBH. During the Hawking radiation, the $\mathrm{BH}$ loses its mass and automatically its entropy, that is its total entropy part by part is transferred to the emitted particles and their correlations.

As shown in [6], at the end of the evaporation we should have only the BH remnant with energy $\omega_{c}$ such that $\omega_{1}+\omega_{2}, \ldots \ldots+\omega_{n}+\omega_{c}=M$. By using the derived formula of the total entropy carried out by radiation [6]

$$
S=-\ln \prod_{i=1}^{n} \Gamma\left(M-\sum_{j=1}^{i-1} \omega_{j} \mid \omega_{i}\right)
$$

one obtains

$$
\begin{aligned}
S & =-\ln \left\{\left(\frac{\omega_{c}}{M}\right)^{\alpha} \exp \left[-\frac{4 \pi}{\Sigma}\left(M-\omega_{c}\right)\right]\right\} \\
& =\frac{4 \pi}{\Sigma} M+\alpha \ln \left(\frac{M}{\omega_{c}}\right)-\frac{4 \pi}{\Sigma} \omega_{c},
\end{aligned}
$$

Here, in order to avoid divergence of the entropy the non-zero $\omega_{c}$ is of vital importance. So, we can infer that the quantum gravity corrected entropy is not valid for mini black holes. In the literature, there are many discussions about the remnant and its implication, see for instance [19]. Constitutively, one can now see that it is a natural result of the quantum gravity effects. Also the inclusion of quantum gravity effects is in accordance with the generalized uncertainty principle, which might cease the complete evaporation of the $\mathrm{BH}$ [20]. Besides this, thinking of the remnant as a non-radiate object having an infinitesimal surface area would not be absurd. From this point of view, in the next section we shall consider the remnant as an extreme LDBH with pointlike horizon and almost zero mass. It will be shown that such a black hole cannot radiate and its temperature would vanish.

Furthermore, the entropy of the remnant can be calculated by applying the Bekenstein's entropy bound [21]. To find it, we reconsider the entropy $S$ carried out by radiation and then follow the following procedure 


$$
\begin{aligned}
S & =\frac{4 \pi}{\Sigma} M+\alpha \ln \left(\frac{\frac{16 \pi M}{\Sigma}}{\frac{16 \pi \omega_{c}}{\Sigma}}\right)-\frac{4 \pi}{\Sigma} \omega_{c} \\
& =\frac{4 \pi}{\Sigma} M+\alpha \ln \left(\frac{16 \pi M}{\Sigma}\right)-\left[\alpha \ln \left(\frac{16 \pi \omega_{c}}{\Sigma}\right)+\frac{4 \pi}{\Sigma} \omega_{c}\right] \\
& =\left(\frac{A_{h}}{4}+\alpha \ln A_{h}\right)-\left[\alpha \ln \left(\frac{16 \pi \omega_{c}}{\Sigma}\right)+\frac{4 \pi}{\Sigma} \omega_{c}\right] \\
& =S_{Q G}-S_{C},
\end{aligned}
$$

One can easily read the remnant's entropy as

$$
S_{C}=\alpha \ln \left(\frac{16 \pi \omega_{c}}{\Sigma}\right)+\frac{4 \pi}{\Sigma} \omega_{c}
$$

From Eq. (30), one can also deduce the conservation of entropy. Clearly, the total entropy of a radiating LDBH $S_{Q G}$ is equal to the entropy of black hole remnant $S_{C}$ plus the entropy carried out by radiation $S$. Being in conform with other recent studies [6, 10], our result shows that the exact spectrum of the Hawking radiation as a tunneling process is not a pure thermal spectrum. This impressive conclusion also implies that information is not lost, and unitarity in QM is restored during the Hawking radiation.

\section{QUANTUM CORRECTED ENTROPY OF THE REMNANT IN HIGHER DI- MENSIONAL LDBHS}

The generic line element for higher dimensional $(N \geq 4)$ static spherically symmetric LDBHs in various theories can be best seen in [12]. In higher dimensions, the metric function $f$ of the LDBHs and the spherical line element are modified to

$$
f=\Sigma r\left[1-\left(\frac{r_{+}}{r}\right)^{\frac{N-2}{2}}\right], \quad d \Omega_{N-2}^{2}=d \theta_{1}^{2}+\sum_{i=2}^{N-3 i-1} \prod_{j=1}^{2} \sin ^{2} \theta_{j} d \theta_{i}^{2},
$$

where $0 \leq \theta_{k} \leq \pi$ with $k=1 . . N-3$, and $0 \leq \theta_{N-2} \leq 2 \pi$. The modified form of the physical constant $\Sigma$ in higher dimensions can also be seen in [12].

As we mentioned before, we would like to set forth the remnant as a higher dimensional LDBH with a pointlike horizon. So, the remnant can be thought as a spacetime with negligible mass. From this point forth, the metric of the remnant can be approximated to a linear dilaton vacuum metric as 


$$
d s^{2}=-\Sigma r d t^{2}+\frac{d r^{2}}{\Sigma r}+R^{2} d \Omega_{N-2}^{2}
$$

One can find the statistical Hawking temperature [22] of this metric as a finite temperature with $T_{H}=\frac{(N-2)}{8 \pi} \Sigma$. But this result is not persuasive since the remnant is massless, clearly a candidate for a non-radiating object, expecting therefore its temperature as zero will be more realistic. To this end, we proceed with a more precise evaluation of the temperature of the remnant from the study of wave scattering in such a spacetime. Metric (33) can be transformed into

$$
d s^{2}=\rho^{2}\left(-d \tau^{2}+d x^{2}+d \Omega_{N-2}^{2}\right)
$$

by

$$
r=e^{\beta x}, \quad t=\frac{\beta}{\Sigma} \tau, \quad \rho=\frac{\beta}{\sqrt{\Sigma}} e^{\frac{\beta}{2} x}
$$

where constant $\beta=A \sqrt{\Sigma}$ (for example, in $4 D$-EMD theory it is 1 [12, 23]). Therefore metric (34) is conformal to the product of $M_{2} \times S_{N-2}$ of a two-dimensional Minkowski space-time with the $(N-2)$-sphere. The massless Klein-Gordon equation

$$
\nabla^{2} \Phi=0
$$

with $\Phi=\rho^{-\left(\frac{N-2}{2}\right)} \Psi$ can be reduced to

$$
-\rho^{\left(\frac{N+2}{2}\right)}\left\{\partial_{\tau \tau}-\partial_{x x}+\left[\frac{\beta(N-2)}{4}\right]^{2}-\nabla_{N-2}^{2}\right\} \Psi=0,
$$

where $\nabla_{N-2}^{2}$ is the $(N-2)$-dimensional Laplace-Beltrami operator with the eigenvalue $-l(l+N-3)[24]$. The reduced Klein-Gordon equation can be rewritten in spherical harmonics with orbital quantum number $l$ as

$$
\nabla_{2}^{2} \Psi_{l}+\mu^{2} \Psi_{l}=0
$$

where the effective mass $\mu$ can be found as

$$
\mu=\left\{\left[\frac{\beta(N-2)}{4}\right]^{2}+l(l+N-3)\right\}^{\frac{1}{2}}
$$


In Eq. (38), $\nabla_{2}^{2}$ is the d'Alembertian operator on $M_{2}$. When viewed from wave propagation aspect, the remnant (34) reduces to the propagation of eigenmodes of a free KleinGordon field in two dimensions with effective mass $\mu$. Conclusively, the remnant cannot radiate and therefore contrary to the calculated $T_{H}=\frac{(N-2)}{8 \pi} \Sigma$ value, its Hawking temperature should be zero.

Finally, we want to find the quantum corrected entropy of the remnant of the LDBHs in an arbitrary dimension. For this reason, by using the surface area of the higher dimensional LDBHs [12]

$$
A_{h}=\frac{16 \pi^{\frac{N-1}{2}}}{(N-2) \Gamma\left(\frac{N-1}{2}\right)} \frac{M}{\Sigma},
$$

one can find the dimension dependent modified area entropy of the BHs as

$$
S_{N Q G}=\frac{A_{h}}{4}+\alpha \ln \left[\frac{16 \pi^{\frac{N-1}{2}}}{(N-2) \Gamma\left(\frac{N-1}{2}\right)} \frac{M}{\Sigma}\right],
$$

and read the dimension dependent quantum corrected tunneling rate

$$
\Gamma_{N} \sim e^{-2 \operatorname{Im}(I)}=e^{-\Delta S_{N Q G}}=\left(1-\frac{\omega}{M}\right)^{\alpha} \exp \left[-\frac{4 \pi^{\frac{N-1}{2}}}{(N-2) \Gamma\left(\frac{N-1}{2}\right)} \frac{\omega}{\Sigma}\right],
$$

We notice that, higher dimensions do not change the correlation between two successive emissions. That is to say, the correlation remains as in $4 \mathrm{D}$ case, see Eq. (27). If we proceed to extend the study to the emission of $n$ particles with energies $\omega_{1}, \omega_{2}, \ldots \ldots, \omega_{n}$, which are successively emitted from the higher dimensional LDBHs, a straightforward calculation will lead us to obtain the dimension dependent entropy carried out by radiation as follows

$$
S_{N}=\frac{4 \pi^{\frac{N-1}{2}}}{(N-2) \Gamma\left(\frac{N-1}{2}\right)} \frac{M}{\Sigma}+\alpha \ln \left(\frac{M}{\omega_{c}}\right)-\frac{4 \pi^{\frac{N-1}{2}}}{(N-2) \Gamma\left(\frac{N-1}{2}\right)} \omega_{c},
$$

This can be rearranged in the form

$$
S_{N}=S_{N Q G}-S_{N C}
$$

where the dimension dependent entropy of the remnant $S_{N C}$ is found to be

$$
S_{N C}=\alpha \ln \left[\frac{16 \pi^{\frac{N-1}{2}}}{(N-2) \Gamma\left(\frac{N-1}{2}\right)} \frac{\omega_{c}}{\Sigma}\right]+\frac{4 \pi^{\frac{N-1}{2}}}{(N-2) \Gamma\left(\frac{N-1}{2}\right)} \omega_{c} .
$$


Eq. (44) is again the conservation of entropy in higher dimensional LDBHs. We conclude that even in the higher dimensional LDBHs information is not lost and the unitarity of QM remains intact.

\section{SUMMARY}

Taking the tunneling formulism and quantum gravity corrected entropy into consideration, the radiation spectra of both $4 D$ and higher dimensional LDBHs are derived. Unlike

our previous study [11], here the probability contains an overall factor with power $\alpha$. As it is shown in this Letter, the role of the factor would be crucial in resolving the information paradox for the LDBHs. By using the tunneling rate with quantum correction, the correlation between successively emitted particles from both $4 D$ and higher dimensional LDBHs is found to be statistically dependent. This nontrivial result proves that information should leak out from the LDBHs during their radiation. When we consider the complete process of the black hole evaporation, the conservation of entropy is obtained for LDBHs in all dimensions and the importance of the black hole remnant proves to be decisive. Absence of the remnant causes entropy divergence, and it is obviously not preferable. Also it is shown that whenever the remnant is modeled as a spacetime with a pointlike horizon - almost zero mass - a higher dimensional linear dilaton vacuum metric can be used to describe it. Using the massless Klein-Gordon equation, it is shown that such a remnant cannot radiate, as expected, and its Hawking temperature would be zero.

In conclusion, the radiation spectrum of the LDBHs deviates from thermal radiation whenever the quantum gravity corrections are taken into consideration. That is, our results are consistent with the unitarity, and show that the information is not lost in the process of Hawking radiation of the LDBHs. Finally, we hope to find charged higher dimensional LDBHs and extent our analysis to those as well.

[1] J.D. Bekenstein, Phys. Rev. D 7, (1973) 2333.

[2] S.W. Hawking, Nature (London) 248, (1974) 30; S.W. Hawking, Commun. Math. Phys. 43, (1975) 199 ; S.W. Hawking, Commun. Math. Phys. 46, (1976) 206, Erratum.

[3] C.E. Shannon, The Bell System Technical Journal 27 (1948) 379-423, 623-656. 
[4] S.W. Hawking, Phys. Rev. D 14 (1976) 2460.

[5] S.W. Hawking, Phys. Rev. D 72 (2005) 084013.

[6] Y.X. Chen, K.N. Shao, Phys. Lett. B 678 (2009) 131.

[7] V.A. Berezin, A. Boyarsky, A.Yu. Neronov, Gravitation \& Cosmology Vol. 5 (1999) 16; K. Srinivasan, T. Padmanabhan, Phys. Rev. D 60 (1999) 024007.

[8] M.K. Parikh and F. Wilczek, Phys. Rev. Lett. 85 (2000) 5042.

[9] A. Boyarsky, A. Neronov, I. Tkachev, Phys. Rev. Lett. 95 (2005) 091301; M. Angheben, M. Nadalimi, L.Vanzo, S. Zerbini, JHEP 0505 (2005) 037; M. Arzano, A.J.M. Medeved, E.C. Vagenas, JHEP 0509 (2005) 037; E.T. Akhemedov, V.A. Akhmedova, D.Singleton, Phys. Lett. B 642 (2006) 124; R. Kerner, R.B. Mann, Phys. Rev. D 73 (2006) 104010; P. Mitra, Phys. Lett. B 648 (2007) 240; R. Banerjee and B.R. Majhi, Phys. Lett. B 662 (2008); E.T. Akhmedov, T. Pilling, D. Singleton, Int. J. Mod. Phys. D 17 (2008) 2453; R. Banerjee, B.R. Majhi, S. Samanta, Phys. Lett. B 662 (2008) 62; R. Banerjee, B.R. Majhi, S. Samanta, Phys. Rev. D 77 (2008) 124035; R. Banerjee, B.R. Majhi, Phys. Rev. D 79 (2009) 064024; R. Banerjee, B.R. Majhi, Phys. Lett. B 675 (2009) 243; R.G. Cai, L.M. Cao, Y.P. Hu, Class. Quant. Grav. 26 (2009) 155018.

[10] B. Zhang, Q.Y. Cai, L. You, M.S. Zhan, Phys. Lett. B 675 (2009) 98.

[11] H. Pasaoglu, I. Sakalli, Int. J. Theor. Phys. 48 (2009), 3415, arXiv:0910.1198 [gr-qc].

[12] S. Mazharimousavi, I. Sakalli, M. Halilsoy, Phys. Lett. B 672 (2009) 177.

[13] J.D. Brown, J.W. York, Phys. Rev. D 47 (1993) 1407.

[14] P. Kraus, F. Wilczek, Nucl. Phys. B 433 (1995) 403.

[15] R. Banerjee, B.R. Majhi, JHEP 0806 (2008) 095.

[16] C. Rovelli, Phys. Rev. Lett. 77 (1996) 3288; A. Strominger, C. Vafa, Phys. Lett. B 379 (1996) 99; S.N. Solodukhin, Phys. Rev. D 57 (1998) 2410; A. Ashtekar, J. Baez, A. Corichi, K. Krasnov, Phys. Rev. Lett. 80 (1998) 904; R.K Kaul, P. Majumdar, Phys. Rev. Lett. 84 (2000) 5255 .

[17] M.A. Nielsen, I.L. Chuang, Quantum Computation and Quantum Information, Cambridge Univ. Press, Cambridge, UK, 2000.

[18] M.K. Parikh, arXiv:hep-th/0402166.

[19] B. Koch, M. Bleicher, S. Hossenfelder, JHEP 10 (2005) 053; X. Li, Phys. Lett. B 647 (2007) 207. 
[20] R.J. Adler, P. Chen, D.J. Santiago, Gen. Relativ. Gravit. 33 (2001) 2101.

[21] J.D. Bekenstein, Phys. Rev. D 23 (1981) 287; J.D. Bekenstein, Phys. Rev. D 49 (1994) 1912.

[22] R.M. Wald, General Relativity, The Un iv. of Chicago Press, Chicago and London, USA and UK, 1984.

[23] G. Clement, D. Gal'tsov, C. Leygnac, Phys. Rev. D 67 (2003) 024012; G. Clément, J.C. Fabris, G.T. Marques, Phys. Lett. B 651 (2007) 54.

[24] D. P. Du and B. Wang, Phys. Rev. D 70 (2004) 064024. 